\title{
Optimal design and analysis of a new thermally actuated microscanner of high precision
}

\author{
W.L. Gambin a and A. Zarzycki \\ Warsaw University of Technology, ul. Sw. A. Boboli 8, 02-525 Warszawa, Poland
}

Received 30 October 2007, Accepted 15 February 2008

\begin{abstract}
A design of a precise, thermally actuated microscanner is proposed, and next, its thermal and mechanical behaviour is considered. The device consists of a micromirror and four thermo-bimorph cantilevers with electric resistors. After the forming process, the mirror assumes an out-of-plane rest position. The process is very simple and compatible with the IC fabrication technique. The mirror is capable of two-dimensional (2D) scans for optical raster imaging. The scanner works both in a non-resonance (for the frame scanning) and resonance mode (for the raster scanning). The high precision of scanning action is achieved due to a special position of the mirror rotation axes with respect to the cantilever beams. The above position assures, that the distance of the mirror centre from the light source is the same during the whole scanning process and the inertial moments of movable parts, as well as the influence of air damping, are minimized. To find the optical angle amplitudes of the mirror, deflections of the cantilevers caused by changes of temperature are determined. The analysis of dynamic temperature distribution enables to determine the thermal cut-off frequency, below which the amplitude of mirror deflections is frequencyindependent. To find the resonance frequencies of the device, the dynamical analysis of the scanner is performed and free vibrations of the considered system are examined.
\end{abstract}

Key words: Microscanners; thermal bimorph actuators; raster scanning system; silicon etching technology; FEM analysis; resonance frequencies; thermal cut-off frequency.

\section{Introduction}

Scanning micromirrors have a wide range of applications. One can find them in devices for imaging, bar-code reading, laser surgery, laser machining, etc. Modern MEMS and MOEMS technologies [1] enable to produce those microscanners smaller and smaller. One can classify microscanners according to their actuation principles. The most common are: electrostatic, piezoelectric, electromagnetic and thermally activated devices.

Microscanners based on electrostatic actuation make the most extensive group of microscanners, because electrostatic actuation is the simplest one [2]. Moreover, these scanners are characterized by very high work frequency advantageous for such devices. Unfortunately, the deflection angle of the mirror actuator is mostly limited. In addition, electrostatic actuation needs quite large voltage. Moreover, it needs a nonlinear control voltage.

Microscanners with piezoelectric actuation have similar properties, although the most important feature of piezoelectric actuation is an almost proportional dependence between the induced strain and the applied electric

\footnotetext{
a Corresponding author:

Wiktor.Gambin@mchtr.pw.edu.pl
}

field [3]. Additional advantages are: high pressure, high power and lower operating voltage during electrostatic actuation. Disadvantages of piezoelectric microscanners are much smaller mirror rotations and more complicated output process.

Micromirrors moved by electromagnetic actuators can realize large scan angle [4]. Movement of the mirror is due to large attraction and repulsion forces generated by the actuator. However, electromagnetic scanners are large because magnetic actuation requires large electromagnets. The large size of the devices leads to great weight of movable parts and, in result, to small operating frequency.

Micromirrors activated thermally, used in the proposed project, have some interesting properties. Thermal actuators, formed as thermo-bimorphic beams, provide large scan angle, nearly linear deflection-power relationship and moderate power consumption. Moreover, fabrication of such microscanners is based on very simple technology. Thermal actuators do not operate with the frequency as high as the electrostatic or piezoelectric actuators, but high enough for some optical applications. Limited frequency is mainly determined by thermal time constants, but it can be improved by using materials with high thermal conductivity. Finally, micromirrors 
a)
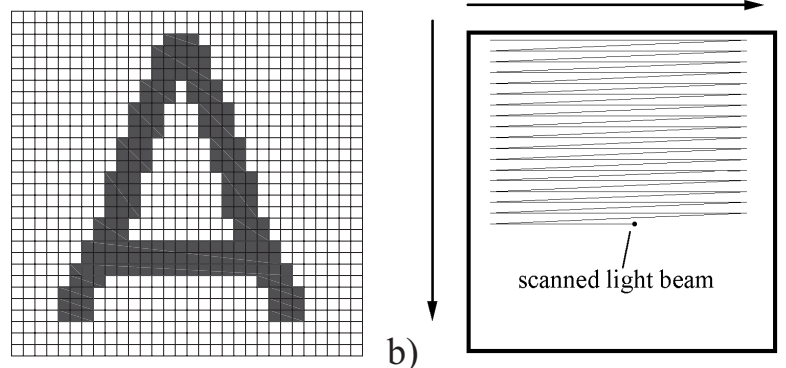

Fig. 1. Display method by raster scanning: a) discrete pixel array, b) light beam motion.

with thermo-bimorphic actuation seem to be very attractive.

\section{Display technique}

Nowadays there are two main display technologies: the two-dimensional discrete pixel arrays [5] and the raster scanning system [6]. There is also the third technique, which combines the above ones [7]. The first technology is based on a micromirror array, where each physical pixel on it responds to a small part of the displayed image. Examples of display technologies using two-dimensional panel include liquid crystal display (LCD) and digital micromirror array, popularly called the digital micromirror display $\left(\mathrm{DMD}^{1}\right)$. The LCD technique is used mostly in different kinds of displays, such as modern television sets, computer monitors etc. The DMD technique is a very popular method of displaying coloured image, and for that reason the producers of multimedia projectors often use it.

The raster scanning system creates a light beam that produces a single bright pixel. The last one is scanned in two dimensions to create an image, in the same manner as a cathode ray tube (CRT) is scanned (Fig. 1). The light beam starts its movement from the left top corner of a screen and moves horizontally (from the left to right). When it reaches the end of the scanned line, the light beam is turned off and moves back to the beginning of the second line. Next, the beam is moving along the second line. That combination of two movements of the beam is repeated till it reaches the end of the screen. After the whole cycle, an image is displayed on the screen. The movement of the light beam along the horizontal line (with a high speed) is called raster scanning, whereas the movement between the lines (with a low speed) is called frame scanning. Both movements are precisely correlated to each other. Raster scanning can be realized in two ways, either by two mirrors scanning in two orthogonal directions or, like in our design, by one two-dimensional scanning mirror. Using only one mirror, the size of the whole device is much more reduced.

As it has been mentioned above, there is the third display technology - the linear-scanning system. It combines

1 DMD is a registered trade mark of Texas Instruments Inc. features of the pixel-array and raster techniques using a one-dimensional array of micromirrors. The micromirror array corresponds to the one-dimensional linear (horizontal or vertical) pixel array of displayed image. In order to display the whole image, the linear pixel array is scanned across the screen, once per frame. The linear-scanning system is widely explored by Silicon Light Machine Inc. The system proposed by them has been called Grating Light Modulator (GLV).

\section{Device design}

Interesting solutions of one-dimensional and twodimensional scanning devices have been described in the book [8]. However, in the case of a two-dimensional microscanner, a certain imperfection is lack of thermal and mechanical separation of perpendicular actuators. It causes undesired twisting and bending of the actuators, and as a consequence, a global distortion of the displayed image. More important is the fact that the distance of the mirror centre from the light source changes during the scanning process. It causes nonuniform resolution of the projected image. Below we propose a solution free from the above drawbacks.

The proposed microscanner is composed of a round micromirror and four thermal-bimorph beams. The beams play the role of actuators, revolving the mirror around two perpendicular axes (Fig. 2a) in two orthogonal planes. One pair of parallel actuators (moving with a high speed and designed for raster scanning) is fixed directly to the mirror, and to a rigid movable frame. Second pair of the bimorph beams (moving at a low speed and designed for frame scanning) is situated perpendicularly to the first one and connects the frame with a stationary silicon substrate.

The actuators are composed of two layers with different coefficients of thermal expansion (CTE) and a thin insulator layer located between them (Fig. 2b). The bottom layer (the passive one) has a lower CTE, whereas the top layer (the active one) has a higher CTE. In our project, the bottom layer has the properties of an electric heater resistor connected with an electric supply. Due to mismatch between the CTE of the materials, the bimorphs after the forming process curl out of plane and make the whole device a 3D-structure.

It is necessary to point out that the described microscanner may be produced using very cheap silicon etching technology [1]. In fact, only two materials are used: silicon and aluminium. As the insulating layer, nitride silicon is sputtered on the bottom layer (with lower CTE) of the actuator, and as a heat resistor is used the same bottom layer made of doped silicon. Due to the special position of the mirror centre with respect to its rotation axes, mirror diameter may be relatively large, what assures a large precision of the displayed image. In the considered project it is assumed as $400 \mu \mathrm{m}$. Thickness of the mirror and its supporting frame is equal to $15 \mu \mathrm{m}$. Total thickness of the bimorph cantilevers is equal to $1.85 \mu \mathrm{m}$, and succeeding layers have the following thickness: doped 
a)
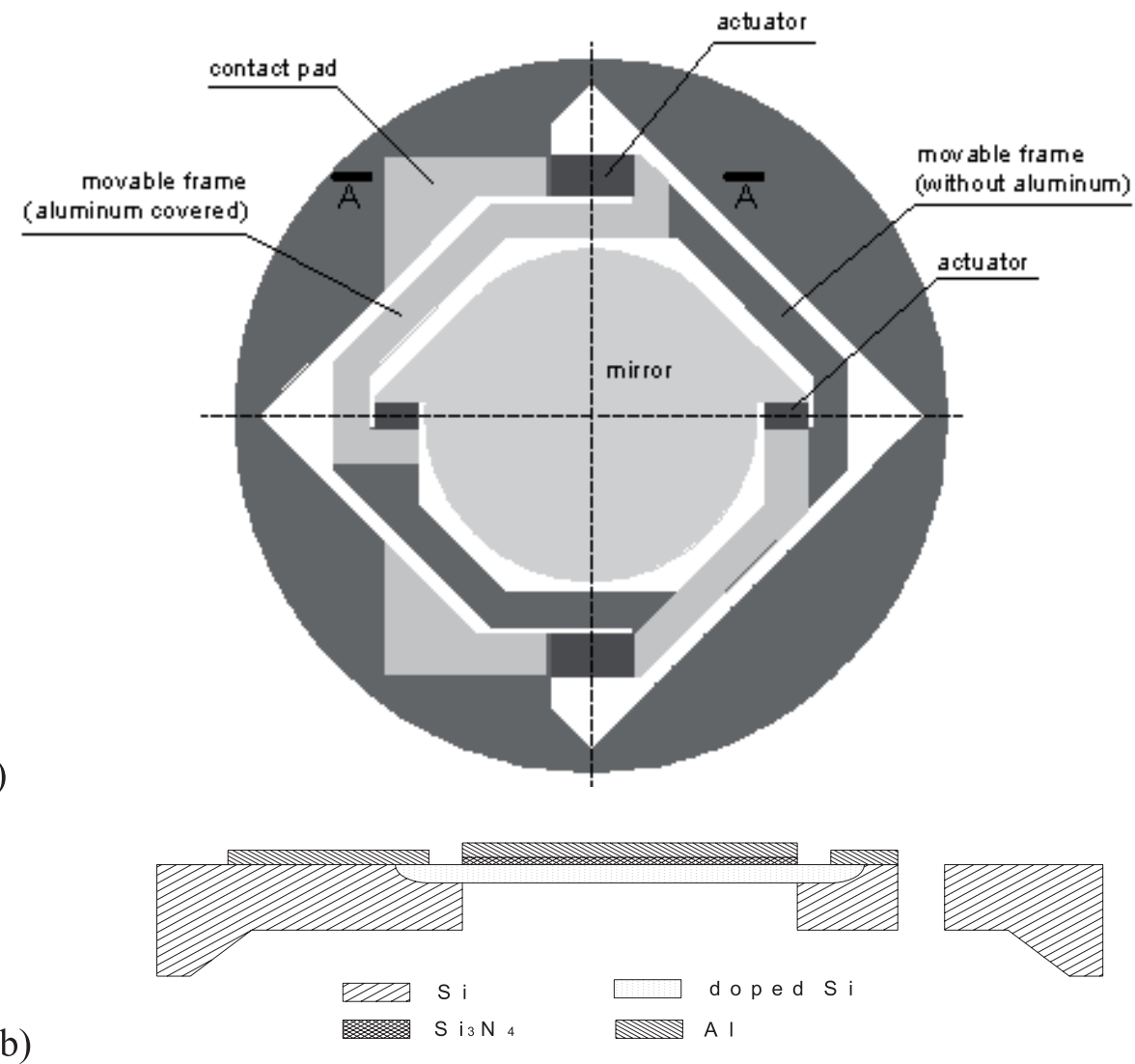

b)

Fig. 2. Proposed microscanner a) planar projection, b) cross-section A-A through the frame and the scanning actuators; materials and layers thickness: Si (frame and mirror) $-15 \mu \mathrm{m}$, doped $\mathrm{Si}$ (passive layer) $-1 \mu \mathrm{m}$, silicon nitride (insulator) $0.15 \mu \mathrm{m}, \mathrm{Al}$ (active layer) $-0.7 \mu \mathrm{m}$.

$\mathrm{Si}$ (passive layer) - $1 \mu \mathrm{m}$; silicon nitride (insulator) $0.15 \mu \mathrm{m} ; \mathrm{Al}$ (active layer) - $0.7 \mu \mathrm{m}$. Width of both types of cantilevers is equal to $50 \mu \mathrm{m}$, and its length is $30 \mu \mathrm{m}$ (for raster scanning) and $100 \mu \mathrm{m}$ (for frame scanning), respectively.

Short description of the microscanner fabrication process is the following. In the first step, the silicon substrate is doped in selected areas. The obtained semi-conductive layer is used, both as an electric heater resistor and a passive layer of actuators. Next, the upper surfaces of the doped areas are covered by $0.15 \mu \mathrm{m}$ insulating layer of $\mathrm{Si}_{3} \mathrm{~N}_{4}$. Afterwards, on the insulating layer, on the upper surface of the mirror and some parts of the moving frame, a thin $(0.7 \mu \mathrm{m})$ aluminium layer is deposited (Fig. 3a). After the cooling process, shrinkage of the aluminium layers of relatively thin actuators causes initial rotation of the mirror (Fig. 3b).

In the room temperature, when electric current is passing through the heater resistor, the temperature of the actuators increases and the structure, initially deflected out of plane, deflects downwards to the substrate plane. Actuators directly connected with the mirror are short and move together with a high resonant frequency, whilst the two others are longer and move together with a low, non-resonant frequency. Due to the fact that the pairs of actuators are perpendicular to each other, and that they move with different frequency, the laser beam reflected by the mirror draws a $2 \mathrm{D}$ raster image on a screen.

As we can see, due to the fact that the cantilevers acting in two different planes are thermally and mechanically separated, one can avoid a global distortion of displayed image. However, the high precision of scanning action is achieved due to a special position of the mirror centre with respect to its rotation axes. In our case, the mirror centre lies on the cross-section of two orthogonal lines joining the centres of cantilevers, (Fig. 2a). The above position assures that the distance of the mirror from the light source is the same during the whole scanning process. Additional advantage is the fact that the inertia moments of movable parts, as well as the influence of air damping, are minimized. It allows to achieve a higher frequency for the frame scanning and a higher resonance frequency for the raster scanning. Higher frequencies result in more accurate projecting image because of greater image refreshing. On the other hand, more precise motion of the mirror causes smaller image distortion. Therefore, keeping a fixed distance between the mirror and the light source during the whole scanning process may significantly improve the displayed image.

To specify the work conditions of the microscanner, it is necessary to determine the dimensions, mechanical 

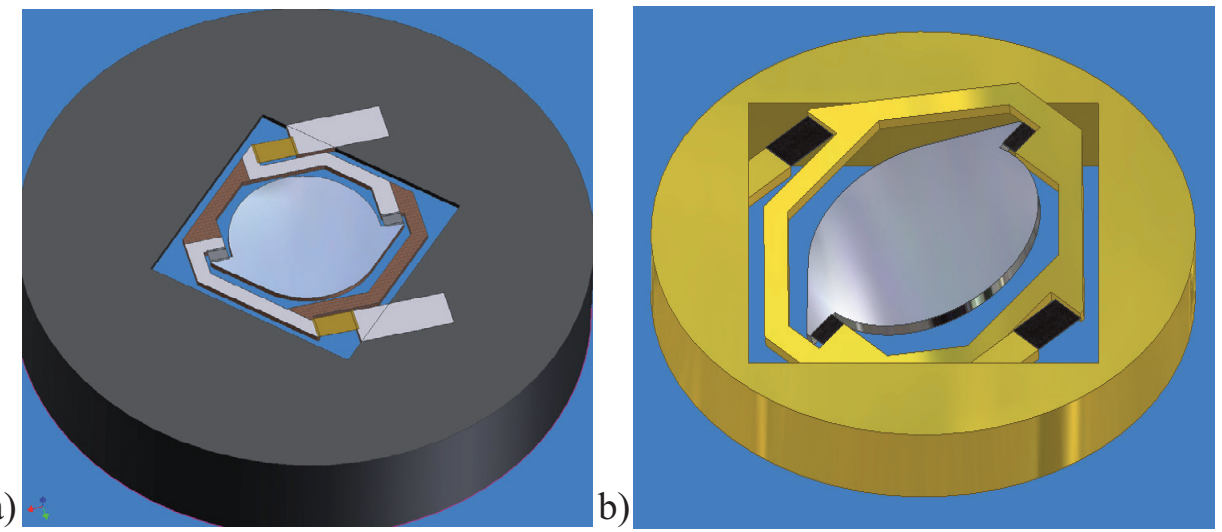

Fig. 3. General view of the microscanner: a) during the forming process, b) after the forming process.

Table 1. Material data.

\begin{tabular}{cccccccc}
\hline Material & $E[\mathrm{GPa}]$ & $C T E\left[\mathrm{~K}^{-1}\right]$ & $\lambda[\mathrm{W} / \mathrm{m} \mathrm{K}]$ & $C[\mathrm{~J} / \mathrm{kg} \mathrm{K}]$ & $\rho\left[\mathrm{kg} / \mathrm{m}^{3}\right]$ & $R[\Omega \mathrm{m}]$ & $\nu$ \\
\hline $\mathrm{Si}$ & 190 & $2.6 \times 10^{-6}$ & 168 & 678 & $2.33 \times 10^{3}$ & $2.3 \times 10^{3}$ & 0.22 \\
Doped Si & 190 & $2.6 \times 10^{-6}$ & 168 & 678 & $2.33 \times 10^{3}$ & $85 \times 10^{-6}$ & 0.22 \\
$\mathrm{Si}_{3} \mathrm{~N}_{4}$ & 270 & $3.3 \times 10^{-6}$ & 30 & 170 & $3.10 \times 10^{3}$ & $10^{14}$ & 0.24 \\
$\mathrm{Al}$ & 69 & $23 \times 10^{-6}$ & 235 & 879 & $2.60 \times 10^{3}$ & $2.65 \times 10^{-8}$ & 0.33 \\
\hline
\end{tabular}

and thermal parameters of the materials, as well as the quantities of exploitation parameters: current, voltage, temperature, resonance and non-resonance frequency and finally the stresses in deformed elements. The assumed mechanical and thermal parameters are presented in Table 1. There are: Young's modulus $(E)$, coefficient of thermal expansion $(C T E)$, thermal conductivity $(\lambda)$, heat capacity $(C)$, density $(\rho)$, resistivity $(R)$ and Poisson's coefficient $(\nu)$.

During the mechanical analysis of the whole device, it is necessary to distinguish the mass and geometrical parameters of the mirror element - working in the resonance mode (Fig. 4a), as well as the mass and geometrical parameters of the element composed of the mirror and a movable frame - working in non-resonance mode (Fig. 4b). Let us introduce a Cartesian system of coordinates, with the centre fixed at the geometrical centre of the mirror. The first element rotating with high speed around the horizontal $x$-axis is divided into two parts, A and $\mathrm{B}$, of the same weight. The mass centres of these parts have coordinates $\left\{x_{\mathrm{A}}, y_{\mathrm{A}}\right\}$ and $\left(x_{\mathrm{B}}, y_{\mathrm{B}}\right\}$. In the same way, the second element rotating with lower speed around the vertical $\mathrm{y}$-axis is divided into parts $\mathrm{C}$ and $\mathrm{D}$ of the same weight. The corresponding mass centres have coordinates $\left\{x_{\mathrm{C}}, y_{\mathrm{C}}\right\}$ and $\left(x_{\mathrm{D}}, y_{\mathrm{D}}\right)$. Notice that the mass centre of the whole device (mirror + frame) lies in the geometrical centre of the mirror.

Owing the above mass distribution, the gravity forces acting on the ends of moving actuators are negligibly small compared with the inertia moments. It considerably reduces the supply of electric power and increases the fundamental resonance frequency of the device. Moreover, the pure bending process of actuators assures a linear dependence between the current voltage and mirror deflection.

\section{Principle of working}

\subsection{Analysis of the "actuator + mirror" system}

To show that distance of the mirror from the light source is the same during the scanning process, let us make the following simple geometrical analysis. Consider a mirror attached to one pair of actuators. Its side view, in the coordinate system $\{x, y\}$, is shown in Figure 5 .

Let the mirror and the cantilevers be located in the plane $y=0$. The beginning and the end of the mirror are at the points $A_{0}$ and $B_{0}$, and the geometrical centre of the mirror - at the point $C_{0}$. Actuators, with the length $L$ each one, are fastened at the point $X_{0}$ and attached to the mirror at the point $Y_{0}$. The distance between the fixing point of the actuators and the centre of the mirror is $a_{0}=L / 2$.

After the forming process, the actuators bend outof-plane and the characteristic points mentioned above: $A_{0}, B_{0}, C_{0}, X_{0}, Y_{0}$, take positions of the points: $A, B, C$, $X=X_{0}, Y$. It is assumed that the pure bending process is due to thermal deformations only, and a new shape of the actuators is a circular arc with the radius $R$ and the centre at the point $O$. The angle $\phi$ (deflection angle) describes the bend of the actuators and the slope of tangent to the arc at the point $Y$.

Below, we will show that the distance between the point $C$ and the plane $y=0$ is smaller than $2 \%$ of the cantilever length $L$, if the angle $\phi$ is smaller than $45^{\circ}$. To do it, denote by $S$ the point of intersection of the moving mirror with the $x$-axis of the coordinate system $\{x, y\}$, and by $a_{S}$ - the distance between $S$ and the fixing point $X=X_{0}$. Let $\delta_{C}$ denote the distance between the centre $C$ of the moving mirror and the point $S$. Notice, that due to symmetry with respect to the $O-S$ axis, the distance $\delta_{C}$ is 
a)

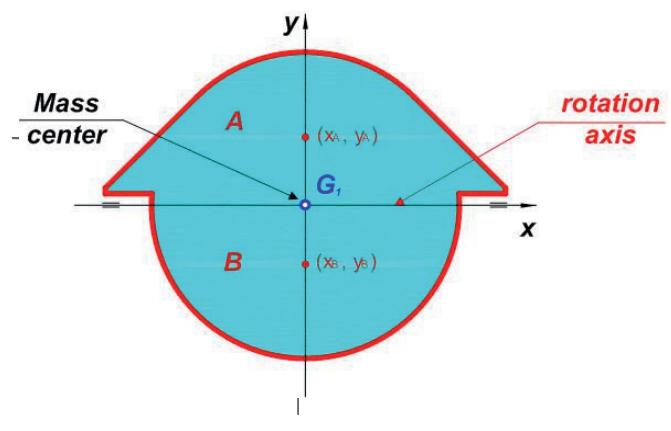

b)

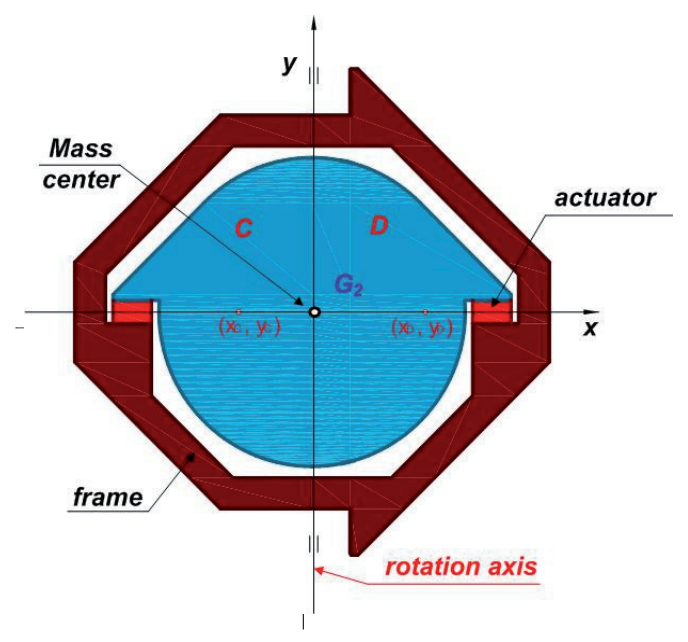

Fig. 4. Mass and geometrical parameters of two elements: a) resonance element (mirror), b) non-resonance element (mirror + frame).

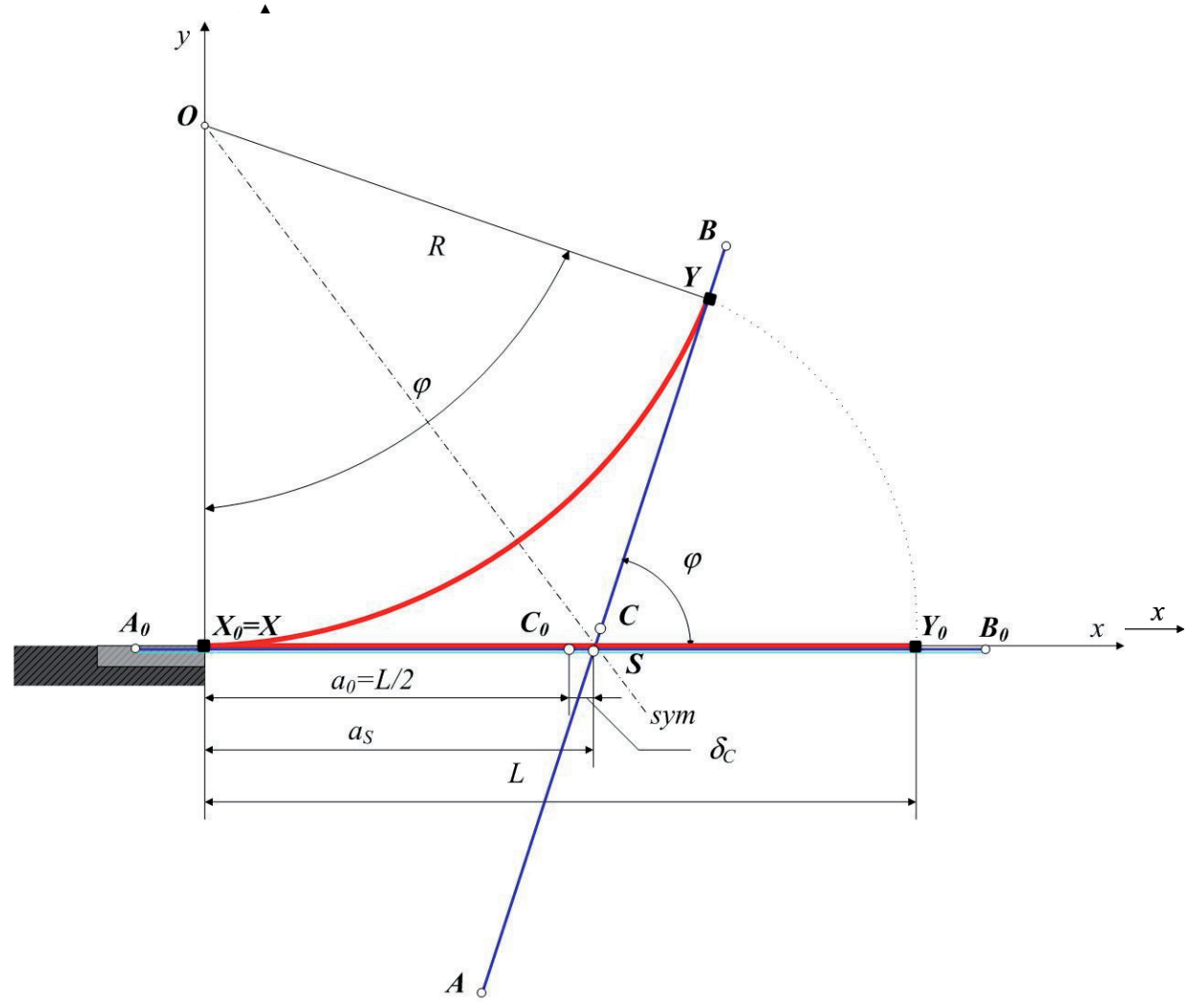

Fig. 5. Geometrical model of the mirror.

the same as the distance between $C_{0}$ and $S$. During the mirror motion, the quantity $\delta_{C}$ changes with the angle $\phi$. Denote by $\delta_{C}^{y}$ the distance between the current position of the mirror centre $C$ and the plane $y=0$. Then, one can write

$$
\delta_{C}^{y}=\delta_{C} \sin \varphi
$$

Assuming that $0 \leqslant \varphi \leqslant \pi / 2$, one can try to estimate the value of $\delta_{C}^{y}$. Because the coordinates of the moving end of the actuator are given by the relations

$$
\left\{\begin{array}{l}
x_{Y}=R \sin \varphi=\frac{L}{\varphi} \sin \varphi \\
y_{Y}=R-R \cos \varphi=\frac{L}{\varphi}(1-\cos \varphi)
\end{array}\right.
$$

the line tangent to the circle at point $Y$ may be described by one of the following equations:

$$
\frac{y-y_{Y}}{x-x_{Y}}=\operatorname{tg} \varphi \text { or } y=x \operatorname{tg} \varphi+\frac{L}{\varphi}(1-\cos \varphi-\sin \varphi \operatorname{tg} \varphi) .
$$




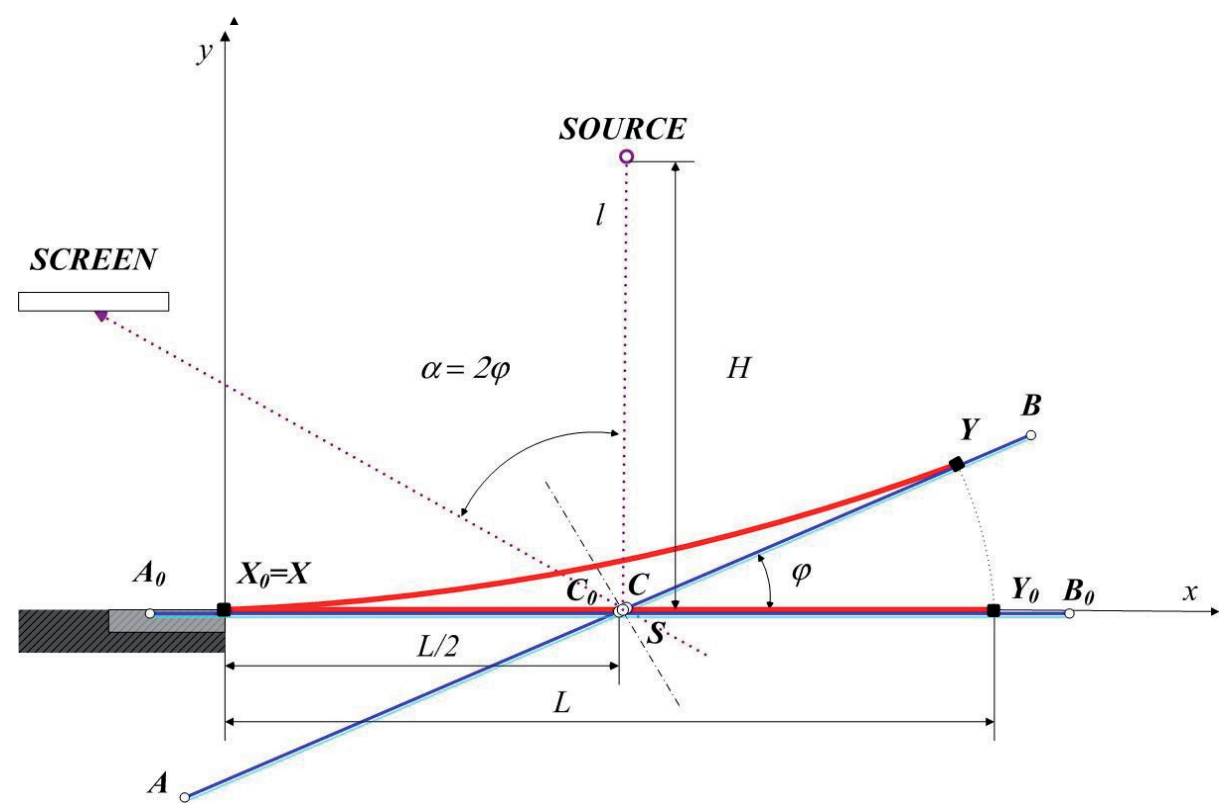

Fig. 6. Geometrical model of the mirror with light source.

Then, the point $S$, which moves along the line $y=0$ during the mirror motion, has the coordinates

$$
\left\{\begin{array}{l}
x_{S}=a_{S}, \\
y_{S}=\frac{L}{\varphi}(1-\cos \varphi-\sin \varphi \operatorname{tg} \varphi)+a_{S} \operatorname{tg} \varphi=0 .
\end{array}\right.
$$

The last relation leads to the formula

$$
a_{S}=\frac{L}{\varphi} \cdot \frac{1-\cos \varphi}{\sin \varphi}, \text { for } 0<\varphi \leqslant \pi / 2 .
$$

Because $\delta_{C}(\varphi)=a_{S}(\varphi)-\frac{1}{2} L$, relation (1) takes the form

$$
\delta_{C}^{y}=L\left(\frac{1-\cos \varphi}{\varphi}-\frac{\sin \varphi}{2}\right) .
$$

From the above rule, one can calculate values of the function $\Delta=100 \% \times \delta_{C}^{y} / L$ for $0^{\circ} \leqslant \varphi \leqslant 45^{\circ}$.

It appears that for $\varphi$ equal to $10^{\circ}, 15^{\circ}, 20^{\circ}, 25^{\circ}, 30^{\circ}$, $35^{\circ}, 40^{\circ}, 45^{\circ}-$ the values of $\Delta$ are equal to: $0.0209 \%$, $0.0744 \%, 0.1795 \%, 0.3418 \%, 0.5873 \%, 0.9264 \%, 1.3720 \%$, $1.9370 \%$, respectively.

Conclusion. For deflection angles from the interval $0 \leqslant \varphi \leqslant 45^{\circ}$, the distance of the mirror centre from the substrate is smaller than $2 \%$ of the actuator length.

\subsection{Analysis of the "actuator + mirror + light source" system}

According to the previous considerations, one can assume that the points $C_{0}, C$ and $S$ at Figure 5 take the same position during a motion of the mirror. It means that the centre of the mirror is at rest when the mirror rotates. A scheme of the considered model supplemented by light source is presented at Figure 6. Light source is stationary and lies on a line $l$ that is parallel to the $y$-axis of the coordinate system and passes through the point $S$. Because the position of the point $S$ practically does not change during the mirror motion, one can assume that the distance from the light source to the point $S$ denoted by $H$ is constant. Denote by $\alpha$ the angle between the light beam incident on the mirror and the reflected one. It is equal twice $\phi$ and is called an optical angle. A light beam incident on the mirror at the centre $S$ is reflected and appears on a screen at the point determined by the optical angle $\alpha$. During the scanning process, the angle $\alpha$ is changing in certain interval. Due to bending of actuators and mirror rotations, optical signals transmitted by the light beam create an image on the screen.

\section{Thermal analysis}

\subsection{Thermal-bimorph beam deflections}

The initial flexure of actuators appears during their forming process. When flat bimorph beams are cooled from the temperature $300{ }^{\circ} \mathrm{C}$ to the room temperature $20^{\circ} \mathrm{C}$, its take the shape of arc with the initial deflexion angle $\varphi_{0}$. To find $\varphi_{0}$, we may use the following rule connecting the change of deflexion angle $\Delta \varphi$ with the change of temperature $\Delta T[9]$ :

$$
\begin{aligned}
\Delta \varphi & =\frac{6 E_{1} E_{2} t_{1} t_{2}\left(t_{1}+t_{2}\right)\left(\alpha_{1}-\alpha_{2}\right)}{4 E_{1} E_{2} t_{1} t_{2}\left(t_{1}+t_{2}\right)^{2}+\left(E_{1} t_{1}^{2}-E_{2} t_{2}^{2}\right)^{2}} \cdot L \cdot \Delta T \\
& =k_{t} \cdot L \cdot \Delta T
\end{aligned}
$$

where $k_{t}\left[\mu \mathrm{m}^{-1} \mathrm{~K}^{-1}\right]$ is the thermal sensitivity of the bimorph, and for $i=1,2, E_{i}-$ the Young modulus, $t_{i}-$ thickness of the layer, $\alpha_{\iota}$ - coefficient of thermal expansion, and $L-$ the length of cantilever. Here, $i=1$ for the active layer (with larger $C T E$ ), and $i=2$, for the passive 


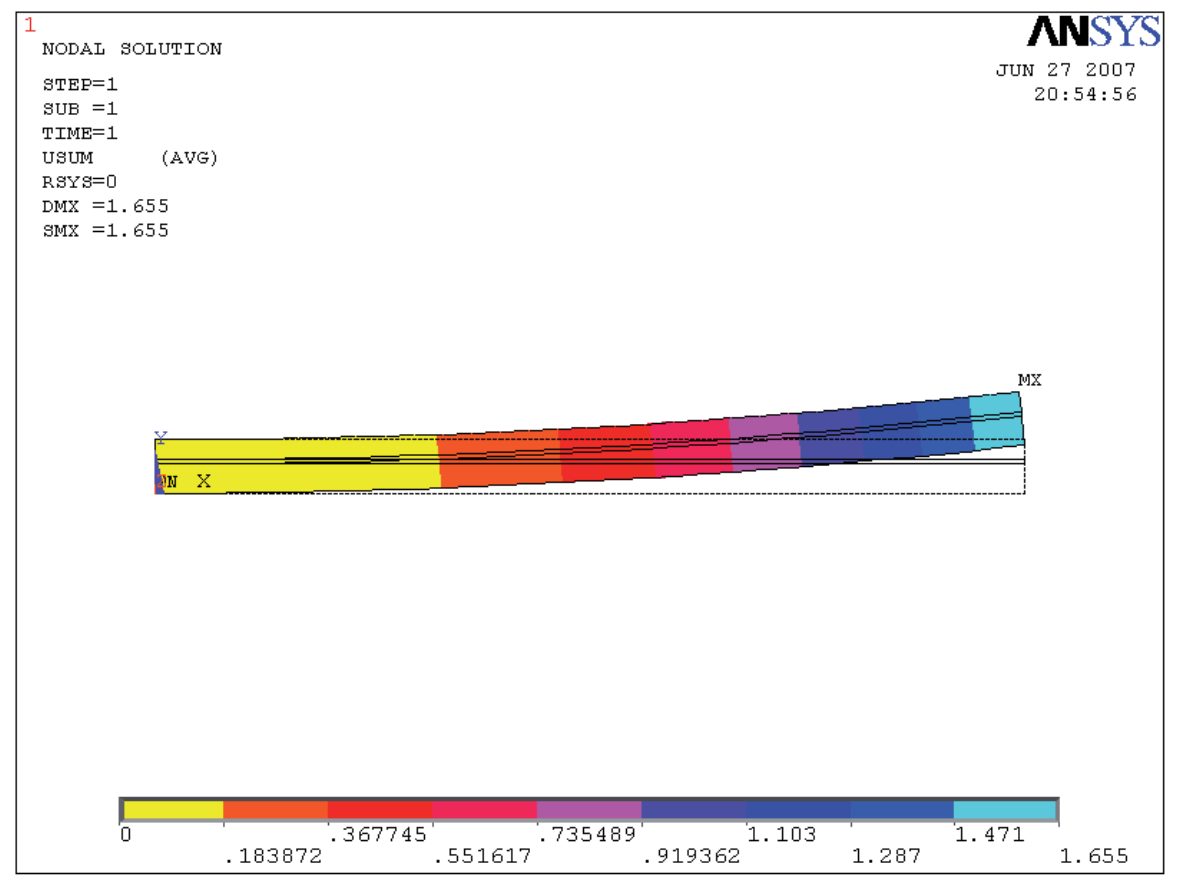

Fig. 7. Initial deflexion of raster scanning actuator.

one (with smaller $C T E$ ). To simplify the calculations, the passive layer is taken as the material composed of doped Si and silicon nitride with thickness $t_{2}=1.15 \mu \mathrm{m}$, with a mean volumetric material parameters. Using the data given in the Table 2 , one can obtain the thermal sensitivity of the actuators $k_{t}=12.19 \mu \mathrm{m}^{-1} \mathrm{~K}^{-1}$. Finally, taking $\Delta T=280{ }^{\circ} \mathrm{C}$, we have - for raster scanning actuators $(L=30 \mu \mathrm{m})$ - the initial deflexion angle $\varphi_{0}=5.87^{\circ}$, and for frame scanning actuators $(L=100 \mu \mathrm{m})$ - the angle $\varphi_{0}=19.56^{\circ}$.

In the above analytical model, actuators were considered as beams composed of two layers with different mechanical properties. Numerical FEM simulations of the shorter actuator, as a beam composed of three layers indicate that insertion of the insulating layer reduces the initial deflexion angle $\varphi_{0}$. Instead of $\varphi_{0}=5.87^{\circ}$, we obtain $\varphi_{0}=5.76^{\circ}$ (Fig. 7). In fact, we have to do with a plate composed of three different layers. During the cooling process such a plate buckles in two perpendicular directions. To verify the influence of the transversal buckling on values of $\Delta \varphi$, numerical simulations of the shorter actuator were performed. In Figure 8, a transversal shrinkage of the plate is shown using the contour map. The corresponding value of the initial deflexion angle $\varphi_{0}$ higher, it is equal to $7.33^{\circ}$.

During the exploitation process, when a driving voltage is applied to the device, the free ends of the actuators oscillate, bending back from their initial positions determined by the angles $\varphi_{0}$, to smaller angles $\varphi=\varphi_{0}-\Delta \varphi$. Using the procedure described above, one can find the values of $\Delta \varphi$.

The values of mechanical angles $\Delta \varphi$ presented in Table 2 are calculated for shorter actuators, which are working in resonance mode with much higher amplitude. The
Table 2. Changes of the deflexion angle for the raster scanning actuators due to changes of temperature.

\begin{tabular}{cccccc}
\hline$\Delta \mathrm{T}=$ & $50{ }^{\circ} \mathrm{C}$ & $100^{\circ} \mathrm{C}$ & $150^{\circ} \mathrm{C}$ & $200^{\circ} \mathrm{C}$ & $250{ }^{\circ} \mathrm{C}$ \\
$\Delta \varphi=$ & $1.07^{\circ}$ & $2.01^{\circ}$ & $3.06^{\circ}$ & $4.11^{\circ}$ & $5.16^{\circ}$ \\
\hline
\end{tabular}

corresponding values $\Delta \varphi$ obtained for longer actuators are four times higher. Equivalent values of optical angles $\Delta \alpha=2 \Delta \varphi$ take magnitudes order of $40^{\circ}$ and are large enough to use the device for image display, in the way shown in Figure 4.

\subsection{Thermal cut-off frequency}

Motion of the microscanner is closely connected with the heat flow and temperature distribution during the actuation process. For frame scanning, one can assume that the temperature in the device is stable and we have to do with a free convection state corresponding to DC drive of the actuators. For raster scanning, when the microscanner is actuated at the resonance frequency, we have to do with a forced heat convection corresponding to $\mathrm{AC}$ mode of beams actuation. In the first case, if changes of the temperature are too fast, the amplitude of the scanner may be frequency-dependent. For that reason, it is necessary to determine the highest frequency exciting the non-resonance vibrations of the scanner. The time constant $\tau$ determining the thermal cut-off frequency of cantilever may be obtained from the following heat transport equation [10]:

$$
\rho \cdot C \cdot \frac{\partial T}{\partial t}=\lambda \cdot \frac{\partial^{2} T}{\partial x^{2}}+P
$$




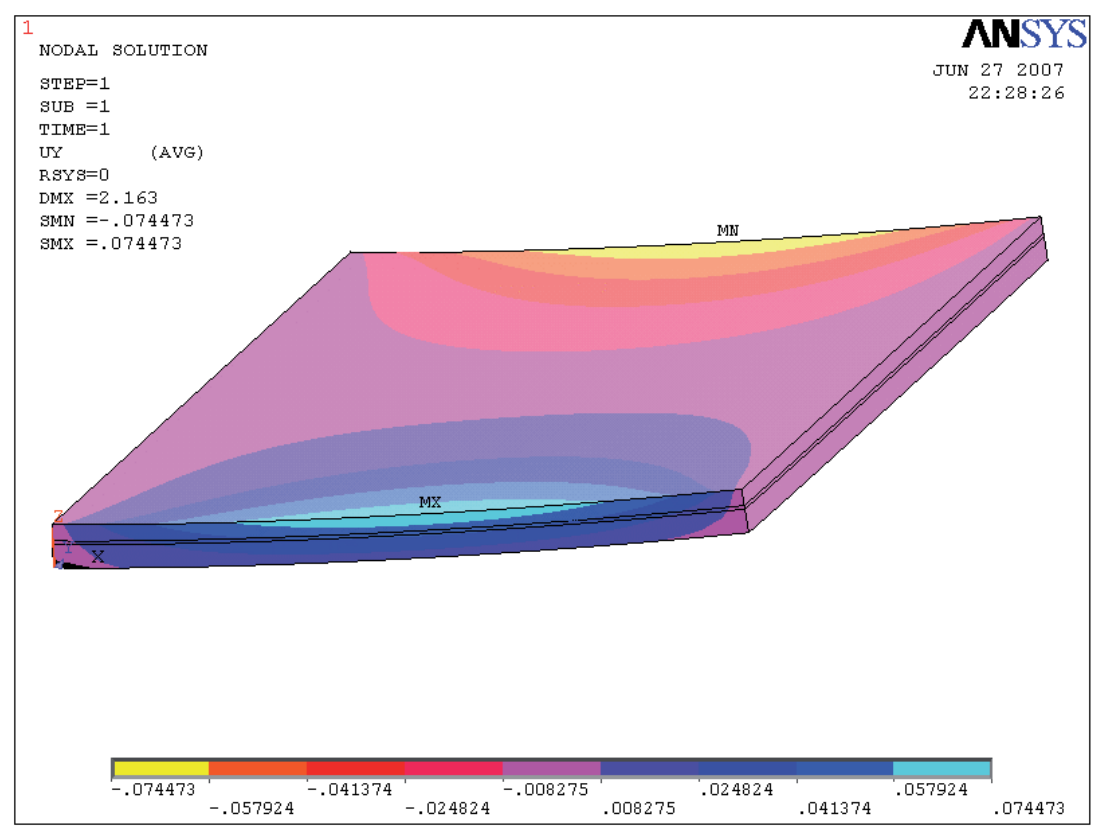

Fig. 8. Transversal shrinkage of raster scanning actuator.

where $x$ is the distance from the fixed point of the cantilever, $\rho, C, \lambda$ - mean parameters of the beam materials, given in Table 1 , and $P$ is the mean electric power dissipated in the heating resistor. At the free end of the beam, when $x=L$ and the maximal stable temperature $T=T_{\max }$, we have

$$
\left.\tau \frac{\partial T}{\partial t}\right|_{x=L}=T_{\max }, \text { where } \tau=\frac{\rho \cdot C}{2 \lambda} L^{2} .
$$

The quantity $\tau$ is the thermal constant time. In our case, mean values of material parameters are the following: $\rho=2.49 \times 10^{3} \mathrm{~kg} / \mathrm{m}^{3}, C=713 \mathrm{~J} / \mathrm{kg} \mathrm{K}, \lambda=$ $182 \mathrm{~W} / \mathrm{m} \mathrm{K}$, and length of the cantilever is $100 \mu \mathrm{m}$. Then, $\tau=0.049 \mathrm{~ms}$. Theoretical value of the thermal cut-off frequency

$$
f_{\text {cut }- \text { off }}=\frac{1}{2 \pi \tau}
$$

gives quite large value $3250 \mathrm{~Hz}$.

\section{Resonance frequency calculations}

\subsection{Analytical model}

To find the fundamental resonance frequency analytically, it is necessary to make some simplifications. Analytical model does not take into account the large deflections of moving cantilevers, and its initial curvature is not taken into account. Also the air dumping and changes of temperature are neglected. Calculations will be performed for the resonance element shown in Figure 4a.

Let us consider, in the coordinate system $\{x, y\}$, a cantilever (actuator), fixed at the point $A$, with a rigid flattened brick element (mirror), attached to its free end $B$ (Fig. 9). Length, width and thickness of the cantilever are denoted by: $l_{a}, b_{a}$, and $t_{a}$, respectively. Length of the loading element is $l_{m}$, and mass is $m_{m}$. Moreover, denote by $J$ the moment of inertia of the brick element with respect to its rotation axis (orthogonal to $\{x, y\}$-plane at the point $B$ ). When the brick element oscillates with rotating motion around its center $C_{0}$, at the end of the cantilever act the force $F$ and the moment $M$ shown in Figure 9 . Because the point $C_{0}$ is practically motionless, we have to do with the case of pure bending considered in the Section 4. Then, one can assume that the force $F$ is equal to zero. If we denote by $\varphi$ the angle between the tangent to the end of the falling beam and the $x$-axis, then

$$
F=0 \text { and } M=J \ddot{\varphi},
$$

where $J=\frac{1}{12} m_{m} l_{m}^{2}$, and $\ddot{\varphi}$ denotes the second timederivative of the function $\varphi$.

To find the fundamental resonance frequency, one can use the general equation of harmonic vibrations: $\boldsymbol{F}+\boldsymbol{K} \cdot \boldsymbol{y}=\mathbf{0},(\boldsymbol{F}$ is the generalized force vector, $\boldsymbol{y}-$ the generalized displacement vector, and $\boldsymbol{K}$ - the stiffness matrix). In our case, the free vibrations are due to the bending moment $M$ only, and the beam stiffness is equal to $E I / l_{a}$. Denoting by $b$ the width of the bimorph cantilever composed of two different materials, we have [9]:

$$
E I=\frac{b}{12} \cdot \frac{E_{1}^{2} t_{1}^{4}+E_{2}^{2} t_{2}^{4}+E_{1} E_{2}\left(4 t_{1}^{2}+6 t_{1} t_{2}+4 t_{2}^{2}\right)}{E_{1} t+E_{2} t_{2}} .
$$

The equation of free harmonic vibrations, takes the form of the following differential equation:

$$
J \ddot{\varphi}+\frac{E I}{l_{a}} \varphi=0
$$

Looking for non-vanishing solution of the form: $\varphi=\varphi_{0} e^{i \omega t}$, we obtain the following form of the 


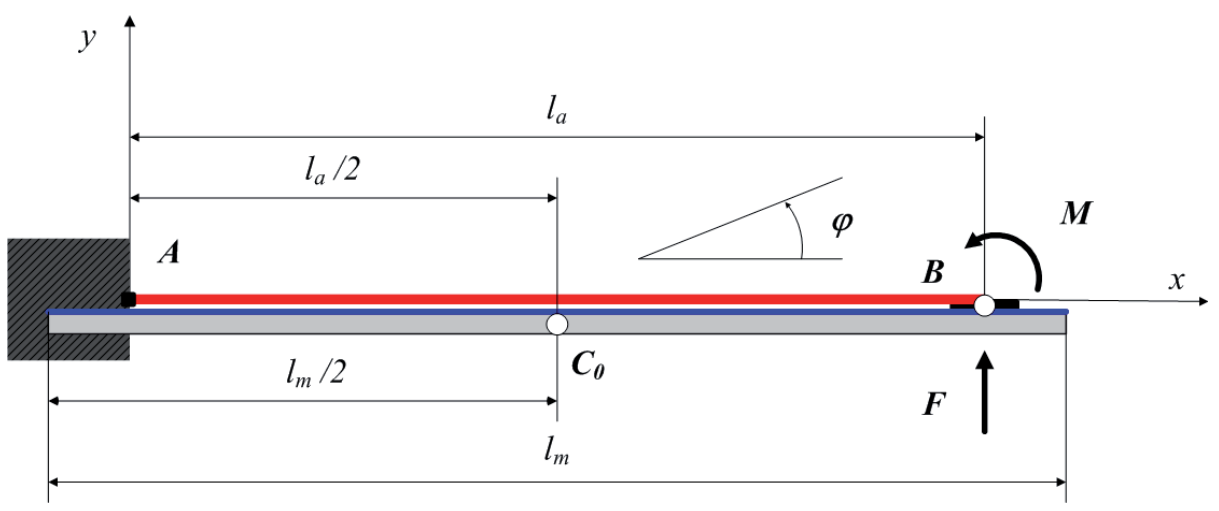

Fig. 9. Cantilever loaded by a mirror attached at point $B$.

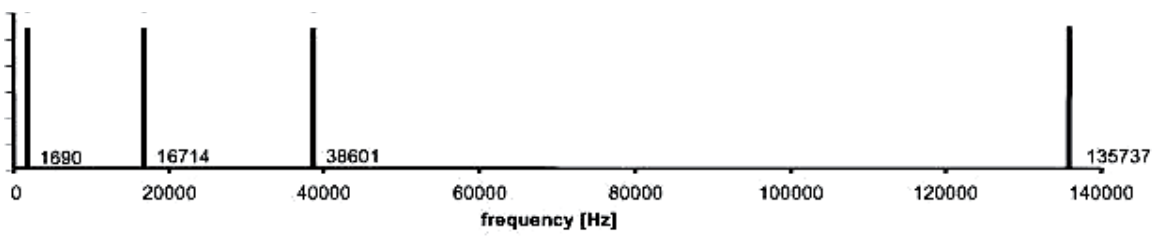

Fig. 10. The first four modes of the resonance frequency of the mirror.
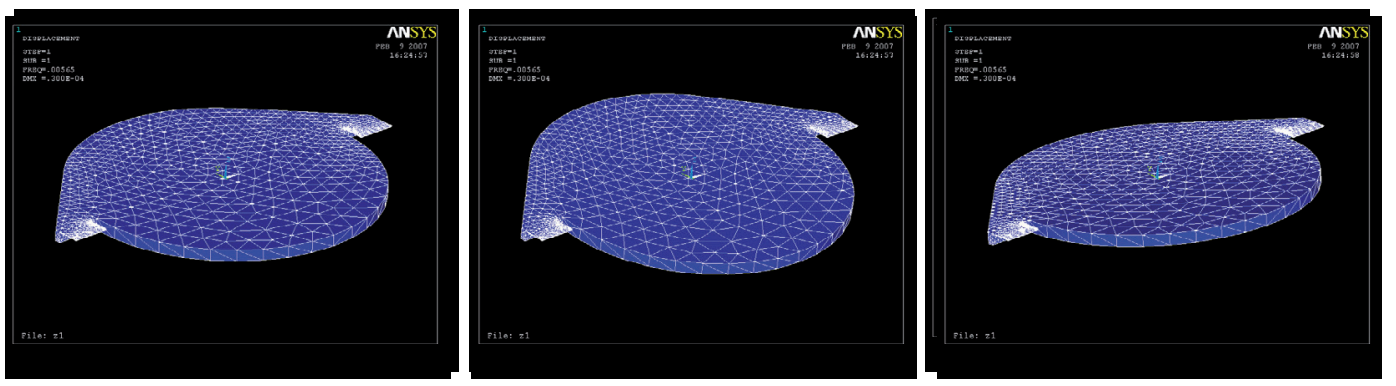

Fig. 11. First mode of the resonance frequency of the mirror.

fundamental natural frequency $\omega_{0}$ :

$$
\omega_{0}=\eta \sqrt{\frac{12 E I}{m_{m} l_{a}^{3}}} \text { where } \eta \equiv \frac{l_{a}}{l_{m}} \text {. }
$$

Using the dimensions and mass density describing the mirror and cantilever, we obtain the fundamental natural frequency $\omega_{0}=9266 \mathrm{rad} / \mathrm{s}$. It corresponds to the frequency $f=1475 \mathrm{~Hz}$. Because the model used is simplified (2D deflections, the rigid mirror without deformations), the real value of $f$ may appear to be smaller. To obtain a more realistic estimation, it is necessary to perform numerical simulations using $3 \mathrm{D}$ model with deformable mirror element.

\subsection{Numerical simulations}

For estimation of the resonance modes of the mirror suspended on the shorter thermo-bimorph actuators, finite element simulations were performed. The obtained five succeeding resonance modes with associated frequencies are: I mode: $1690 \mathrm{~Hz}$, II mode: $16719 \mathrm{~Hz}$, III mode: $38601 \mathrm{~Hz}$, IV mode: $135137 \mathrm{~Hz}$ and V mode: $135760 \mathrm{~Hz}$.
The first mode of oscillations is due to rotations of the mirror around the axis crossing the free end of the cantilever (Fig. 11). Quite interesting is the second mode, when the mirror is moving without rotations, remaining in parallel horizontal planes. The higher modes are connected with twisted forms of the mirror plate. Notice that the obtained frequency $\omega_{0}=1052 \mathrm{~Hz}$, described by mode $\mathrm{I}$, is large enough for the scanning process.

\section{Conclusions}

Detailed analysis of the solutions presented in the book [8] leads to a new solution of the thermally actuated microscanner of high precision. The most important conclusions are the following:

- thermal and mechanical separation of actuators working in different planes enables to avoid their twisting and, in consequence, it is possible to avoid global distortions of the displayed image;

- locations of mass centres of the moving parts of the device in the geometrical centre of the mirror, suppress the inertial forces acting on the rotating elements and, in consequence, it considerably diminishes the 
power supply, increases fundamental resonance frequency and assures a linear dependence between the current voltage and mirror deflection;

- special location of the geometrical center of the mirror with respect to actuators enables to keep a constant distance from the light source to the center of the mirror and, in consequence, it assures a uniform, high resolution of the displayed image.

\section{References}

1. M. Madou, Fundamentals of Microfabrication (CRC Press, Boca Radon, Florida, USA 1997)

2. K.E. Petersen, IBMJ. Res. Develop. 24, 631 (1980)

3. K. Yamada, T. Kuriyuma, A novel asymmetric silicon micro-mirror for optical beam scanning display, in Proceedings of MEMS'98 (Heidelberg, Germany, pp. $110-115,1998)$

4. R.A. Miller, W.G. Burr, SPIE 2687, 47 (1996)

5. K.E. Petersen, Appl. Phys. Lett. 31, 521 (1977)
6. M.-H. Kiang, O. Solgaard, K.Y. Lau, J. Microelectromechanical Systems 7, 27 (1998)

7. R.W. Corrigan, Scanned linear architecture improves laser projectors, Laser Focus World (1999), pp. 169-172

8. G. Lammel, S. Schweizer, Ph. Renaud, Optical Microscanners and Microspectrometers using Thermal Bimorph Actuators (Kluwer Academic Publishers, Boston, 2002)

9. S. Timoshenko, J. Opt. Soc. America 11, 233 (1925)

10. F.P. Incropera, D.P. DeWitt, Fundamentals of Heat and Mass Transfer (John Wiley \& Sons, New York, 1996)

11. W.L. Gambin, A. Zarzycki, Optimal design of a new thermally actuated microscanner of high precision, First International Conference on Multidisciplinary Design Optimisation and Application, April 17-20, (2007) EDP Sciences, ISBN 978-27598-0023-0

12. W.L. Gambin, A. Zarzycki, Thermal and dynamical analysis of new microscanner behaviour, First International Conference on Multidisciplinary Design Optimisation and Application, April 17-20, (2007) EDP Sciences, ISBN 97827598-0023-0 